\title{
Über die relativen Theilungspotenzen einiger Embryonalzellen.
}

\author{
Von \\ R. S. Bergh. \\ Mit Tafel XIX und 3 Textfiguren. \\ Eingegangen am 13. Mai 1895. \\ Vorbemerkungen.
}

Uber das im Titel dieser Abhandlung genannte Thema weiß man bis jetzt eigentlich gar nichts, bis auf das, was sich auf die Furchungsprocesse vieler Thiere bezieht. Über diesen letzteren Gegenstand ist schon hinreichend bekannt, wie in vielen Fällen einige Zellen durch besonders lebhafte Theilungsfähigkeit anderen (ihren Schwesterzellen) gegenüber ausgezeichnet sind, und in vielen Fällen hat man den Verlanf der Theilungen so genau beschrieben, dass hieraus zu entnehmen ist, wie groß die relativen Theilungspotenzen der einzelnen Zellen sind. - Ganz anders verhält es sich mit den späteren Embryonalstadien der Thiere. Hier hat man nirgendswo uiber die relativen Theilungspotenzen der verschiedenen Zellen etwas ermitteln können, wohl aus dem Mangel einer passenden Methode. Es wäre in der That in den meisten Fällen eine höchst schwierige - mit der gegenwärtigen Methodik wohl unmögliche Aufgabe, zu bestimmen, wie groß die Theilungsfähigkeit der einzelnen Zellen in den verschiedenen Regionen der Keimscheibe z. B. irgend eines Wirbelthiereies ist.

Es ist nun eine sehr bemerkenswerthe Erscheinung, dass bei vielen Anneliden und Crustaceen das Wachsthum des Keimstreifens mittels einer größeren oder geringeren Anzahl von hinten gelegenen sog. Urzellen oder Teloblasten, welche durch eine längere Periode immerfort kleinere Zellen in der Richtung nach vorn sprossen, 
stattfindet (es ist dies ein ähnlicher Vorgang wie das den Botanikern schon lange bei vielen, namentlich niederen Pflanzen bekannte Wachsthum mittels Scheitelzellen). Und zwar kann man in dem Keimstreifen sowohl von Anneliden wie von Crustaceen äußere und innere Urzellen und von diesen gesprosste kleinere Zellen unterscheiden, welche letztere eine regelmäßige, reihenförmige Anordnung zeigen. Besonders in dem äußeren, oberfächlichen Theil des Keimstreifens der Crustaceen tritt diese Regelmäßigkeit ganz besonders deutlich hervor. Es giebt nämlich hier meistens eine sehr große - bei versehiedenen Formen verschiedene -- Anzahl von Teloblasten, und die ron ihnen gesprossten kleineren Zellen stehen äußerst regelmäßig zu Längs- und Querreihen zusammeng*eordnet, so dass der ganze hintere Theil des Keimstreifens ein karrirtes Aussehen hat, zwei Systeme von Linien unterscheiden lässt.

Diese Thatsachen: dass bei vielen Crustaceen and Anneliden äußere und innere Urzellen sich finden, sowie dass die von ihnen gesprossten kleineren Zellen in regelmäßige Längs- und Querreihen geordnet sind, waren schon einer Anzahl von Autoren des letzten Decenniums bekannt; doch beschränkten sich fast alle Verfasser darauf, diese Befunde festzustellen ohne die Sache weiter zu verfolgen und ihre Ursachen zu ergründen. So Patten 1), ReichenBach $^{2}$ ), Claus ${ }^{3}$ ), Nusbaum ${ }^{4}$ ) u. A. Nur in meinen Arbeiten über Mysis und Gammarus ${ }^{5}$ ) wurde ein erster Versuch gemacht, in die Mechanik des Wachsthums durch Untersuchung der Stellung und Anordnung der Mitosen einzudringen, und diesen Versuch habe ich in der vorliegenden Arbeit etwas weiter geführt. Es gelang mir in meinen früheren Arbeiten namentlich für Mysis festzustellen, zunächst dass alle Mitosen in dem hinteren Theil des Keimstreifens - der durch die regelmäßige Anordnung der Zellen charakterisirt ist - gleiche Stellung einnehmen, indem nämlich die Äquatorialplatten immer quer (senkrecht zur Längsachse des Embryos) ge-

1) On the origin of Vertebrates from Arachnids. Quart. journ. of micr. sc. Vol. 31. 1890. p. 371.

2) Studien zur Entwickelungsgeschichte des Flusskrebses. Abhandl. d. Senckend. Naturf. Gesellsch. Bd. 14. 1886.

3) Untersuchnngen über die Organisation von Branchipus und Artemia Arbeiten a. d. zool. Institut Wien. Bd. 6. 1886.

4) Materialien zur Embryogenie und Histogenie der Isopoden (in polnischer Sprache). Schriften der Akademie zu Krakau. Bd. 25. 1893.

5) Beiträge zar Embryologie der Crustaceen. I-II. Zoolog. Jahrbücher. Bd. 6-7. Abth. f. Anat. u. Ontog. d. Thiere. 1893. 
stellt sind, so dass jede Zelle in eine vordere und eine hintere Tochterzelle zerfällt, and ferner dass in jeder Querreihe die Theilungen von der Mitte nach den Seiten hin fortschreiten, so dass häufig eine im lateralen Theil einfache Querreihe nach der Medianlinie zu in zweie zerlegt ist.

Als ich nun diese Untersuchungen wieder aufnahm, wurde mir eine neue Erscheinung klar, welche ich früher nicht beachtet hatte: nämlich dass im hinteren Theil des Keimstreifens die Mitosen konstant in gewissen Zonen, in bestimmten, von den Urzellen aus numerirten Querreihen auftreten, in anderen dagegen immer fehlen, und es gelang mir, ans diesem Verhalten in sehr einfacher Weise das Verhältnis der Theilungspotenz der Urzellen zu derjenigen der kleineren Zellen abzuleiten. Ich fing die Untersuchung an den Embryonen einiger Land-Isopoden (Oniscus, Armadillidium) an, nahm danach wieder die alten Objekte (Mysis und Gammarus) anf und ging schließlich an die Untersuchang des Keimstreifens einiger Anneliden (Criodritus, Lumbricus). In dieser Reihenfolge sind die Ergebnisse auch unten mitgetheilt.

\section{Oniscus murarius und Armadillidium vulgare.}

Da bei beiden eben genannten Arten gänzlich übereinstimmende Verhältnisse gefunden wurden, sind sie unter einer Rubrik gemeinsam abgehandelt.

Die Zahl der Urzellen des äußeren (ektodermalen) Keimstreifens schwankt bei beiden Formen zwischen 21 und 25; immer ist eine median gelegene Zelle vorhanden. Vor den Urzellen finden sich die von jenen gesprossten und - entsprechend ihrem Ursprunge - in regelmäBige Längs- und Querreihen gestellten kleineren Zellen. Die Zahl der Querreihen beträgt in dem roll ausgebildeten Zustand des Keimstreifens - etwa von dem der auf Ligia sich beziehenden Fig. 4 von J. Nosbaum (1.c.) entsprechenden Stadium bis zu dem Zustand, wo die reihenförmige Anordnung undeutlich wird -- 19-21; vor der vordersten Querreihe wird die Anordnung der Zellen eine unregelmäßige. Sehr selten ist die Zahl der deutlich unterscheidbaren Querreihen größer als 21; das Maximum von 25 Querreihen sah ich nur einmal. Die Zahl der Längsreihen entspricht in dem hintersten Theil des Keimstreifens derjenigen der Urzellen, ist also 21-25; in dem vorderen Theil des Keimstreifens ist sie aber eine kleinere, weil die am meisten lateral gelegenen 
Längsreihen kurz vor ihrem Ursprunge undeutlich werden, indem ibre Zellen sich den angrenzenden gewöhnlichen Ektodermzellen beimischen und ihre reguläre Anordnung aufgeben. In roll ausgebildeten Keimstreifen (mit etwa 20 Querreihen) konnten im vordersten Theil des Keimstreifens nur etwa 15-17, in juingeren Stadien (mit 12-13 Querreihen) sogar nur 11 Längsreihen unterschieden werden. Beispielsweise bestand in einem Falle an der einen Seite die äußerste Längsreihe nur aus 4, die zweitäußerste aus 8, die dritte aus 12, die vierte aus 18, die tünfte aus 19 Zellen, und an der anderen

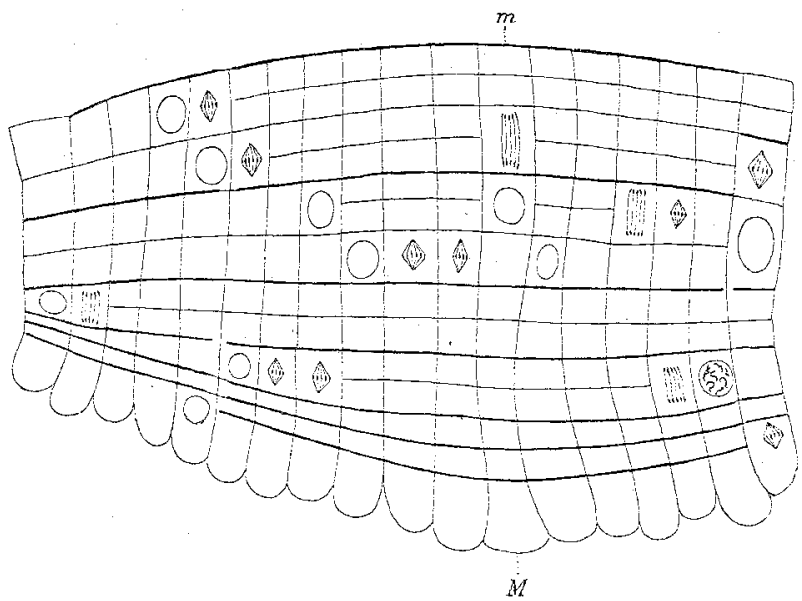

Fig. 1.

Stück ảes hinteren Theils des Keimstreifens eines Embryos von Oniscus mararius (auf dem Stad. 4 von Nusbaum). D. Oc. 1 (Zerss). Die Mitosen sind eingezeichnet und in den Zellen, die sich demnächst zur Theilung anschicken, sind die Kerne angegeben. Vgl. iibrigens den Text.

Seite waren die Verhältnisse ganz ähnliche (die Zahl der Urzellen war hier etwa 25). Wenn daher im Vorhergehenden und im Folgenden von der Zahl der Querreihen die Rede ist, bezieht sich das immer auf den mittleren, vollständigen Theil des Keimstreifens; in den seitlichen Theilen ist die Zahl der Querreihen immer eine kleinere.

Dass in den seitlichen Theilen des Keimstreifens weniger Querreihen als im mittleren Theil vorhanden sind, .beruht nicht allein darauf, dass die seitlich gelegenen Keimstreifzellen vorn sich den gewöhnlichen Ektodermzellen beimischen, sondern zugleich auf einem anderen Umstand, nämlich darauf, dass die mittleren Urzellen den seitlich gelegenen in der Produktion der kleineren Zellen voraus- 
geeilt sind, so dass die aus jenen entspringenden Zellreihen vollzähliger als die von diesen ausgehenden sind. Dies ist namentlich in den älteren Stadien sehr dentlich zu beobachten; weniger macht sich der Unterschied in den jüngeren Stadien des Keimstreifens bemerkbar. In mehreren der beigegebenen Figuren ist dies sehr dentlich erkennbar: sowohl in den Textfiguren 1 und 2, wie auch in Fig: 4 (Taf. XIX). In den beiden ersteren erkennt man, wie an einem bestimmten Punkt die seitlichen Urzellen plötzlich stärker nach vorn vorspringen als die mehr mediad gelegenen. Dies ist

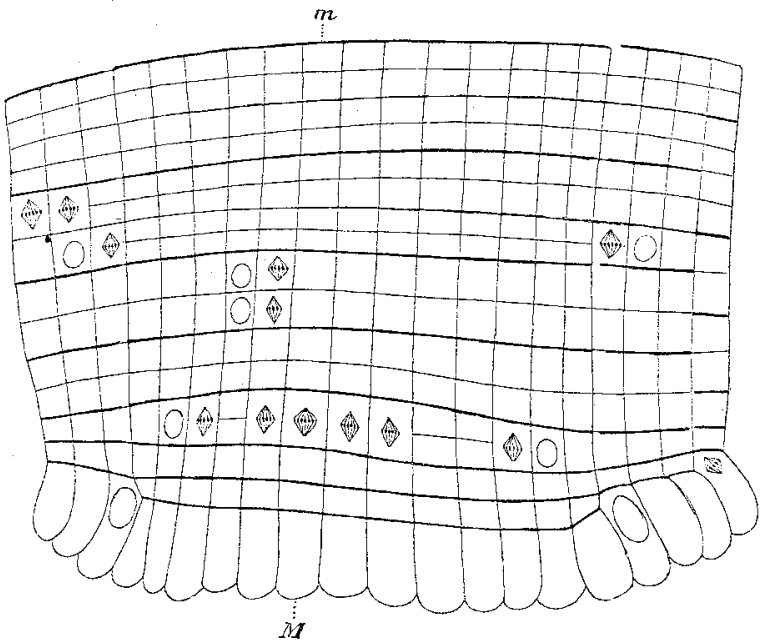

Fig. 2.

Der größte Theil des Keimstreifens eines Embryos von Oniscus murarius, wenig jünger als Stad. 4 von Nusbaux. In derselben Weise wie Fig. 1 ausgeführt. D. Oc. 1.

z. B. in Fig. 1 an der einen Seite mit der Urzelle VI, an der anderen mit der Urzelle VII der Fall, in Fig. 2 mit der Urzelle VI an der einen, VIII an der anderen Seite; in Fig. 4 (Tafel XIX) springt erstens die Zelle $\mathrm{V}$ vor; einen weiteren Vorsprung bildet Zelle VIII und einen noch weiteren Zelle $\mathrm{X}^{1}$ ). Und für jeden solchen Vorsprung fehlt eine Zelle in der betreffenden und in den weiteren, lateral sich anschließenden Längsreihen: so sehen wir in letztge-

1) Die Längsreihen und die Urzellen sind im Folgenden immer mit römischen Zahlen bezeichnet, so dass I die der medianen Reihe zunächst gelegene bezeichnet. Die Querreihen sind mit arabischen Zahlen bezeichnet, so dass 1 die hinterste, jüngste, den Urzellen direkt anliegende bezeichnet. 
nannter Figur die Reihen I--IV aus 7, V-VII aus 6, VIII-IX nur aus 5 (resp. 4, da eine Theilung der kleineren Zellen noch nicht vollzogen ist) bestehen; die Zellen X-XII sind noch weiter zurïck. Zugleich erkennt man, wie die seitlichen Keimstreifzellen vorn in gewöhnliche Ektodermzellen übergehen.

Ebenso wie die Urzellen sich nur in einer bestimmten Richtung inäqual theilen, indem sich vorn kleinere Zellen von ihnen $a b-$ schnüren, ebenso finden die ersten Theilungen, welchen die kleineren Zellen sich unterziehen, nur in einer bestimmten Richtung statt, indem die Theilungsebenen immer senkrecht zur Längsachse der Embryonalanlage stehen; dabei sind die Theilungen äqual (und natiurlich antiklin). Es werden hier bestimmte Regeln firr die Aufeinanderfolge der Theilungen eingehalten und zwar eine Längsreihenregel und eine Querreihenregel, wie ich mich kurz ausdrücken möchte. Erstere ist im vollentwickelten Keimstreifen so streng durchgeführt, dass man fast von einem Gesetz reden könnte; im vollentwickelten Keimstreifen mit etwa 20 Querreihen fand ich nie und nimmer Ausnahmen. Diese Längsreihenregel für den vollentwickelten Keimstreifen ist folgende: Zelle 1 und 2 (von der Urzelle aus gerechnet) sind nie in Theilung begriffen; dagegen theilen sich die Zellen konstant, wenn sie durch zwei jüngere Schwesterzellen von der Urzelle getrennt sind, jede in eine vordere und eine hintere Zelle (3 und 4). Nun wird eine neue Zelle von der Urzelle gesprosst; die Zellen 3 und 4 werden also nun zu Nr. 4 und 5; dann theilt sich die nächste, nachgeschobene Zelle (3), die jetzt durch zwei jüngere Schwesterzellen von der Urzelle getrennt ist, und die Urzelle knospt gleich danach wieder eine Zelle; durch die letzten Vorgänge sind dann Nr. 4 und 5 (die Descendenten der ursprïnglichen Zelle 3, von der wir ausgingen) in $\mathrm{Nr} .6$ und 7 rerwandelt worden, und in dieser Lage schicken sie sich nun wieder zur Theilnng an. Besser als jede wörtliche Beschreibung erläutern aber dieses die Textfiguren 1 und 2. Dieselben sind halbschematisch; doch möchte ich hervorheben, dass sie beide nach Präparaten, sogar mit dem Prisma entworfen sind; die einzigen Schematisirungen bestehen erstens darin, dass nicht jede kleine Begrenzungslinie der Zellen angegeben wurde (wie in den Figuren auf der Tafel XIX), sondern dass die Längsund Querreihen dort durch ununterbrochene Grenzlinien geschieden sind; und zweitens darin, dass einige Linien stärker, andere schwächer gezeichnet sind; letzteres Verfahren lässt die Zusammengehörigkeit der verschiedenen Zellen nach Generationen erkennen. 
Ein Jeder, der diese Figuren anfmerksam betrachtet, wird erkennen, wie die oben gegebene Regel überall und ausnahmslos zutrifft: die drittjüngste Zelle ist schon getheilt oder schickt sich zur Theilung an, und ebenso die Zellen, die durch 6 oder 7 Zellen von der Urzelle entfernt sind; dagegen werden in den Zellen 1, 2, 5 und 6 niemals Mitosen angetroffen (dabei beachte man die bald zu erwähnende Querreihenregel).

Von den zwei aus der Theilung einer Mutterzelle entstandenen Tochterzellen theilt sich meistens erst die vordere und sehr bald danach die hintere; doch gehen bisweilen die Theilungen beider fast gleichzeitig yor sich. Beide Fälle, häufiger jedoch der erste, kommen in den Textfiguren 1-2 zum Ausdruck.

Als Resultat der zweiten Theilung erhalten wir also anstatt zwei, vier hinter einander liegende Zellen, und in Folge dessen sieht man die Zellen im vorderen Theil des Keimstreifens in den Längsreihen zu Gruppen von vieren zusammengeordnet; die genetische Zusammengehörigkeit der daraus resultirenden Gruppen von Querreihen ist je in den Textfiguren durch die dicken Linien angedentet. Die Zellen ruhen nun wieder vor der nächsten Theilung und demgemäß findet man in den Zellen 9-12 (von der Urzelle aus gezählt) keine Mitosen; wohl kann man aber solche in den Zellen 13-16 treffen. Tch habe hier nicht mit solcher Konstanz wie in den vorhin erwähnten Fällen die Mitosen nachweisen können; es scheint, dass schon hier der Verlauf etwas weniger regulär wird, and dass in einigen Fällen die Zellen ihre frühere Theilungsgeschwindigkeit beibehalten, während in anderen Fällen die Theilungen schon mit geringerer Geschwindigkeit auf einander folgen, so dass man erst weiter vorn (in den Reihen 17-20) die Mitosen findet. Sobald die Zellen - abgesehen von solchen reinen Ausnahmefällen, in welchen eine größere Anzahl Querreihen als 20-21 vorhanden sind - durch 19--20 jüngere Zellen von der Urzelle entfernt sind, fangen die Theilungen an, unregelmäßiger vor sich zu gehen: die Theilungsebenen stehen hier nach sehr verschiedenen Richtungen. Oft sieht man gerade vor der vordersten Querreihe eine ganze Anzahl Mitosen, deren (antiklinen) Theilungsebenen parallel zur Längsachse des Embryos sind; in anderen Fällen ist die Anordnung der Theilungsebenen eine ganz unregelmäßige. Damit geht natürlich die reguläre Anordnung der Zellen in Längs- und Querreihen verloren.

Jede der kleineren Zellen macht also, während sie sich im 
regulär angeordneten Theil des Keimstreifens befindet, drei Theilungen durch. Und es ist leicht ersichtlich, dass wenigstens während der ersten Periode ihrer Existenz ihre Theilungspotenz fast dreimal so klein ist, wie die der Urzellen. Denn bevor eine solche neuentstandene, kleinere Zelle sich zur Theilung anschickt, hat die Urzelle inzwischen zwei neue Zellen gesprosst, und gleich nach der Theilung jener theilt sie sich wieder. Und bei der zweiten Theilung wird genau der gleiche Rhythmus eingehalten. Bei der dritten konnte dies nicht mit gleicher Konstanz nachgewiesen werden; vielleicht nimmt hier die Geschwindigkeit des Rhythmus für viele der kleineren Zellen ab. Mitosen sind im vorderen Theil des (regulär angeordneten Theils des) Keimstreifens bei Weitem nicht so häufig, wie im hinteren Theil.

Zur Erkenntis der Zusammengehörigkeit der Zellen nach Generationen und zur Ermittelung ihres etwaigen Alters seit der letzten Theilung helfen auch die Form und Größe derselben sehr gut. Denn die kürzlich aus einer Theilung hervorgegangenen Zellen sind klein und von vorn und hinten abgeplattet, wogegen die sich zur Theilung anschickenden Zellen viel größer sind und etwa dieselbe Quer- und Längsdimension haben. So sieht man meistens (z. B. in Textfigur 2) recht dentlich, wie die eben von den Urzellen gesprossten Zellen der Querreihe 1 kleiner sind, als ihre älteren Geschwister in der Querreihe 2; diese sind wiederum kleiner als die sich theilenden oder sich zur Theilung anschickenden in der Querreihe 3. Und dergleichen findet man auch weiter vorn. Eine Querreihe, deren Zellen in größerer Anzahl sich zur Theilung vorbereiten oder schon in Mitose begriffen sind, wird oft sowohl vorn wie hinten vorgewölbt (bikonvex). Auf die Bedeutung der eben erwähñten Erscheinungen für die mechanischen Ausführungen ïber die Zelltheilungen und deren Aufeinanderfolge habe ich übrigens schon an anderer Stelle aufmerksam gemacht ${ }^{1}$.

Die Querreihenregel, d. h. die Regel von der Aufeinanderfolge der Zelltheilungen in den Querreihen, ist dieselbe, wie ich sie schon früher für Mysis und Gammarus angegeben habe, nämlich diese: dass die Zelltheilungen von den medianen nach den Seitentheilen fortschreiten; doch erleidet diese Regel bei Oniscus und Armadillidium weit zahlreichere Ausnahmen, als bei den obgenannten Formen; worauf dieses beruht, vermag ich nicht zu sagen. Zunächst ist zu

1) Vorlesungen über allgemeine Embryologie. Wiesbaden. 1895. pag. 94 ff. 
beachten, dass bei allen diesen Formen die in der unpaaren, medianen Längsreihe gelegenen Zellen keineswegs immer Schritt halten mit den seitlichen, wie für Gammarus schon früher angegeben wurde; nach der aufgestellten Regel sollte man denken, jene Zellen seien in der Theilung diesen immer vorans; das ist aber bei Weitem nicht der Fall. Aber auch in den seitlichen Längsreihen trifft man durchans. nicht selten Ausuahmen, so dass eine mehr seitlich gelegene Zelle einer Querreihe oft getheilt ist, während die der Medianlinie näher gelegenen noch ungetheilt oder in Theilung begriffen sind. Die Fig. 1 und 4 (Taf. XIX) sowie Textfigur 2 lassen dies deutlich erkennen, während Textfigur 1 das normale Verhalten wiedergiebt. Besonders wenn die Theilungen sehr rasch auf einander folgen, sind solche UnregelmäBigkeiten sehr häufig (so in Textfigur 2, Querreihe 3, in welcher gleichzeitig 6 Mitosen vorhanden sind; ebenso in Fig. 4 Taf. XIX, wo in der Querreihe 3 drei Zellen noch in Theilung, während zwei soeben die Theilung vollendet haben). Immerhin bleibt in den bei Weitem häufigsten Fällen die oben aufgestellte Regel zu Recht bestehen.

In den jüngeren Stadien sind für den lateralen Theil des Keimstreifens ganz dieselben Verhältnisse leicht nachzuweisen; dagegen sind im medianen Theil ein wenig geänderte Verhältnisse zu erkennen, indem nämlich die Thätigkeit der Teloblasten im Verhältnis zu derjenigen der kleineren Zellen hier eine noch intensivere ist, als in den späteren Stadien. Besonders in solchen jungen Stadien, in welchen nur 6-7 Querreihen von den Teloblasten gesprosst sind, beobachtet man solche Relationen, wie sie in den Fig. 2 und 3 (Taf. XIX) wiedergegeben sind. In Fig. 2 sieht man rechts in der Längsreihe $I$ die Zelle 4 in Theilung begriffen; alle nahe liegenden Zellen derselben Querreihe sind schon in zweie zerlegt. Die Urzellen haben hier (jede) drei jüngere Zellen gesprosst, bevor die vorderste der abgebildeten Querreihen in die Theilung eintrat; hier beobachtet man fast als Regel die Irregularität, dass die der medianen Längsreihe anliegenden Zellen später als die mehr lateral gelegenen sich zur Theilung anschicken. In Fig. 3 sieht man gleichfalls, wie die Zelle 4 der mehr medial gelegenen Längsreihe in die Mitose eingetreten ist, während der lateral zunächst liegende Teloblast im Begriff ist, eine junge Zelle zu sprossen; nach Vollendung der $\mathrm{Ab}$ schnürung derselben wird die Zelle 4 ihrer Längsreihe erst in die Theilung eintreten.

Aus meinen Beobachtungen geht also hervor, dass die 
Theilungspotenz der Urzellen oder Teloblasten in fertig gebildeten - das heißt: schon etwa 20 Querreihen enthaltenden - Keimstreifen gegenüber derjenigen der kleineren Zellen als Einheit $<3$, in dem der Medianlinie genäherten Theil des jüngeren Keimstreifens aber noch gröber: $>3$ ist, und dass die ersten Theilungen der kleineren Zellen nach einer bestimmten Richtung and nach einem bestimmten Rhythmus stattfinden; erst wenn sie dureh ca. 20 Zellen von ihrer Urzelle getrennt sind, wird der Verlanf ihrer Theilungen unregelmäBiger.

Wenn oben gesagt wurde, dass in der Querreihe 1 und 2 nie and nimmer Theilungen vorkommen, so ist das richtig; doch können solche in vereinzelten Fällen dadurch vorgetäuscht werden, dass einzelne Zellen durch starken Druck der Umgebungen voribergehend in die Tiefe geschoben werden. Einen solchen Fall habe ich in Fig. 5 mittels des Zeichenprismas dargestellt; die nebenstehende Fig. 5 a ist eine Abstraktion and stellt dar, wie die mittlere der drei Zellreihen im Sagittalschnitt anssehen miisste. In dieser Reihe scheint in der That bei oberfächlicher Untersuchung die Zelle 2 in Theilung begriffen; nur bei sehr genaner Einstellung erkennt man, wie zwischen ihr und der Zelle 1 eine sehr schmale, trennende Zelle liegt, deren Hauptmasse mit dem Kern tiefer, zum größten Theile unterhalb der sich theilenden Zelle liegt. Diese letztere ist also in der That Zelle 3. Wirkliche Theilungen in den Querreiben 1 und 2 habe ich, wie gesagt, nie gefunden, und Fälle wie Fig. 5 sind überdies sebr selten.

\section{Mysis $\mathrm{sp.}$}

Schon in meiner ersten Arbeit über die Entwicklung dieses Thieres habe ich die in der Einleitung erwähnten Regeln für die Zelltheilungen im Keimstreifen anfgestellt. Doch habe ich es damals noch nicht versucht, die relativen Größen der Theilungspotenzen der Teloblasten und der von ihnen produzirten, kleineren Zellen festzustellen. Nachdem nun fur die Land-Isopoden das Verständnis hierfür gewonnen war, nahm ich wieder die Mysis-Embryonen vor, und zu meiner nicht geringen Überraschung stellte sich heraus, dass die Verhältnisse in bezüglicher Hinsicht fast ganz dieselben, wie bei den Land-Isopoden waren. In dem dem Ausschliupfen nahe stehenden Nauplius-Stadium (mit umgebogenem Schwanze) findet man 
die Theilungen der kleineren Zellen des (ektodermalen) Keimstreifens niemals in den zwei unmittelbar vor den Urzellen befindlichen Querreihen; die erste Theilung der von jenen abgeschnürten kleineren Zellen findet, wie auch in den späteren Stadien statt, während sie in der dritten Querreihe gelegen sind. Dieses Verhalten ist in Fig. 6 sehr deutlich zum Ausdruck gebracht, in der auch die ganz normale Reihenfolge der Theilungen in den Querreihen hervortritt: die beiden Zellen I rechts und links haben sich schon soeben getheilt; die beiden Zellen II sind in der Dyasterphase und die weiter seitwärts gelegenen sind noch ungetheilt; die Theilung schreitet also von den mittleren nach den seitlichen Theilen fort. Nur die mediane Zellreihe hält - wie bei Gammarus and den Land-Isopoden - ihren eigenen Rhythmus der Theilung ein. Ausnahmen ron dieser Querreihenregel sind bei Mysis anffallend selten, weit seltener als bei den Isopoden.

In den jüngeren Nauplius-Stadien, d. h. in solchen mit noch

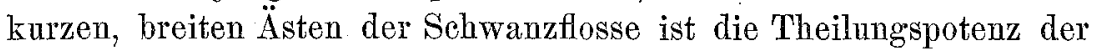
Urzellen im Verhältnis zu derjenigen der kleineren Zellen eine noch größere. Es lässt sich dies daraus schließen, dass man in diesen Stadien nicht in den Zellen, die durch zwei, sondern in jenen, welche durch drei juingere Schwesterzellen von ihren Urzellen entfernt sind, die Mitosen findet. Fig. 7 giebt das Verhalten in diesen Phasen wieder: die Zelle 4 IV rechts ist in Theilung begriffen, die Zelle $4 \mathrm{~V}$ noch ungetheilt; links ist noch keine der betreffenden Zellen (4 IV und 4 V) getheilt; dagegen sind an beiden Seiten 4 I-4 III schon fertig getheilt. Diese Theilungen in der vierten Querreihe habe ich in diesen jungen Phasen recht häufig beobachtet. Einmal wurde ganz genau das gleiche Bild wie Fig. 3 (Armadillidium) gesehen.

Es geht aus diesen Beobachtungen mit Sicherheit hervor, dass die Theilungspotenz der Teloblasten sich zu derjenigen der kleineren, von ihren gesprossten Zellen in älteren Stadien wie $<3: 1$, in jüngeren Stadien wie $>3: 1$ verhält. Also ganz wie bei Oniscus und Armadillidium.

Bei Mysis scheint die Theilungspotenz der kleineren Zellen schon nach der ersten Theilung abzunehmen. Bei den Onisciden ging $j a$ die zweite Theilung nach gleichem rhythmischem Intervall wie die erste vor sich und lagen demgemäß diese Mitosen in den Reihen 6-7 oder 7-8. Bei Mysis kommen aber diese Mitosen erst weiter vorn im Keimstreifen vor. In Fig. 6 sieht man vor den Teloblasten 8-9 Querreihen; aber Mitosen finden sich nur in der 
dritten Reihe; auch ist aus den GröBenverhältnissen ersichtlich, dass in den vordersten Reihen noch keine Theilungen stattgefunden haben, sondern dass die Zellen in den vordersten Reihen sich erst zur Theilung: anschicken. Die Umbiegungsstelle des Schwanzes liegt meistens an der Grenze der Reihen 7-8, 8-9 oder 9-10 (gleich vor der vordersten Reihe der Fig. 6 war der Schwanz abgebrochen). Nun findet man in dem umgebogenen Schwanztheil niemals Mitosen anßer in der dritten Querreihe (resp. in der vierten bei den juingeren Embryonen) und vor der Umbiegungsstelle gewöhnlich auch nicht in der allerhintersten Reihe, wohl aber ein wenig weiter vorn. Eine bestimmte Regel für die Reihenfolge der Theilungen in den Längsreihen habe ich hier nicht nachweisen können; möglich, dass eine solche doch existirt und einem späteren Forscher erkennbar wird; dagegen gilt auch hier die Querreihenregel vollauf. - Im mittleren Theil des vollansgebildeten Keimstreifens finden sich meistens 24-25 dentlich unterscheidbare Querreihen; vor der Reihe 24 oder 25 wird die Anordnung der Zellen eine mehr unregelmäBige.

\section{Gammarus pulex.}

Die Eier dieses Thieres wurden mit in die Untersuchung hineingezogen, um zu prïfen, wie die Verhältnisse bei einem Thiere liegen, dessen Keimstreifen in seinem oberflächlichen (ektodermalen) Theil ohne Teloblasten wächst. Es ist ja eine auffallende Erscheinung, auf die ich schon in meiner früheren Arbeit hingewiesen habe, dass trotz des Fehlens der Teloblasten die Zellen des Ektoderm-Keimstreifens in Längs- und Querreihen sehr regelmäßig angeordnet sind (ähnlich wie unter den Anneliden bei Rhynchelmis, bei welcher die Urzellen ganz fruh verschwinden). Es erfolgt demgemäß das Wachsthum zunächst durch Theilung der schon in den Keimstreifen eingetretenen Zellen, dann aber auch dadurch, dass von hinten allmählich gewöhnliche Blastodermzellen sich zu Längs- und Querreihen - als Fortsetzung der schon vorhandenen - zusammenordnen und so den Keimstreifen verstärken helfen. Es geschieht dies, ohne dass diese hinten nachrïckenden Zellen durch besonders große Theilungspotenz ausgezeichnet sind, denn in der betreffenden Region sind Mitosen entschieden seltener als in dem Keimstreifen selbst zu finden.

Es giebt also bei der Bildung des Keimstreifens von Gammarus keine durch besonders große Theilungspotenz ausgezeichnete Zellen. 
Nichtsdestoweniger lassen sich einige Übereinstimmungen im Wachsthum mit Mysis und mit den Land-Isopoden nachweisen. Zunächst finden wir, jedenfalls im mittleren Theil des Keimstreifens dieselbe »Querreihenregel " für die Richtung und Anfeinanderfolge der Theilungen: in den Querreihen schreitet die Vermehrung der Zellen von der Medianebene nach den Seiten hinaus fort und alle Theilungen gehen in derselben Richtung vor sich, ganz wie bei Mysis (im seitlichen und hinteren Theil des Keimstreifens kommen auch andere Richtungen und Folgen der Theilungen vor, was wohI mit der eigenthiumlichen Drehung des Keimstreifens zusammenhängt; man vergleiche hieriiber meine frühere Arbeit). Im voll ausgebildeten Keimstreifen - d. h. wenn eine große Zahl von Läng'sund Querreihen sichtbar geworden sind, etwa den Stadien Fig. 3 und 6 meiner früheren Arbeit entsprechend - lässt sich auch eine gewisse RegelmäBigkeit fuir das Auftreten der ersten Theilungen in den Längsreihen feststellen. Wie schon frïher mitgetheilt, geht die mediane Zellreihe hinten in eine unregelmäßige, breitere Zellmasse über und demgemäß divergiren hier die etwas weiter hinten noch zu erkennenden, seitlichen Längsreihen. Betrachten wir nun diese Stelle,

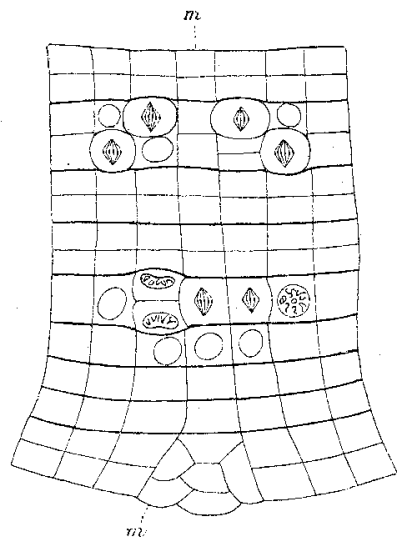

Fig. 3.

Stück des hinteren Theils des Keimstreifens von Gammanus pulex, in derselben Weise wie Textfigur 1 und 2 ausgeführt. D. 0 c. 1. wo die mediane Zellreihe in die unregelmäßige Zellmasse übergeht, als einen fixen Punkt, so ist es auffallend, dass in den ersten drei Querreihen niemals Mitosen zu finden sind, dass sie dagegen in der vierten oder fünften Reihe von dem erwähnten Punkt aus sehr. häufig sind (vgl. Textfigur 3 und Taf. XIX Fig. 8) und zwar schien es mir, dass in den jüngeren Stadien (Taf. XIX Fig. 8) die Mitosen besonders in der fünften, in der späteren (Textfigur 3 ) in der vierten Querreihe vorhanden sind. Vor der durch Mitosen ausgezeichneten Reihe liegen immer zwei Reihen, die sich durch die Formverhältnisse ihrer Zellen als durch Theilung einer einzigen entstanden manifestiren. Würden wir nun die erste vor dem er- 
wähnten Punkt liegende Querreihe der Reihe der Teloblasten bei Mysis und den Isopoden gleichstellen - d. h. nur in topographischer Hinsicht - , so wäre, wie leicht ersichtlich, die Übereinstimmung eine sebr vollständige. - In ganz jungen Stadien konnte keine solche Regelmäßigkeit nachgewiesen werden.

Die zweite Theilung der Zellen im Keimstreifen findet erst statt, nachdem die Zellen in der Reihe 9 oder 10 vorgeschoben worden sind, und zwar kann die zweite Theilung bald in der vorderen, bald in der hinteren der beiden aus der ersten Theilang: stammenden Tochterzellen friher eintreten, wie aus Textfigur 3 ersichtlich ${ }^{1}$.

\section{Lumbricus, äulsere Keimstreifen.}

Meine Untersuchungen ïber diesen Gegenstand haben nicht zu hinreichend befriedigenden Ergebnissen geführt. Denn trotzdem ich etwa die Keimstreifen von 20 Embryonen untersuchte, fand ich nur. in einem Falle eine Theilung in der Nähe der Urzelle, nämlich in der sechsten Zelle; es war dies in der slateralen Zellreihe " oder in der Reihe IV). Anßerdem habe ich in meiner früheren Arbeit iiber diesen Gegenstand ${ }^{2}$ ) zwei Figuren dargestellt, in denen solche

1) Aus den Änßerungen, die Herr Delua Valle (Gammarini. Fauna und Flora des Golfes von Neapel. 20. Monographie. 1893. p. 886] über meine frühere Arbeit macht, scheint hervorzugehen, dass es ihm nicht erwünscht war, dass während des Druckes seines großen Werkes eine kleine Arbeit erschien, durch welche ein großer Theil seiner Ergebnisse erschüttert wurde. Hätte DecLA VALLE den eigenthimlichen Drehungsvorgang beachtet und auch durchsichtig: gemachte Flächenpräparate studirt, so wäre wohl seine Beschreibung der Keimblätterbildung und der Entstehung der Muskelplatten richtiger ausgefalien (er lässt das Entoderm durch Abspaltung des Blastoderms und die Muskelplatten durch regellose Auswanderung amöboider Zellen, hauptsächlich aus dem Ektoderm entstehen). Was den letzteren Punkt betrifft, so wurde schon in meiner früheren Arbeit mitgetheilt, dass die betreffenden Bildungen ihren Ursprang aus Teloblasten haben und Streifen innerhalb des Ektoderm-Keimstreifens bilden; doch vermochte ich damals nicht die Zahl jener festzustellen. Dieses ist mir jetzt durch erneate Untersachangen gelungen und zwar stellte sich dadurch eine erfrenliche Übereinstimmung mit Mysis und mit den Isopoden heraus: auch bei Ganmarus finden sich jederseits vier Teloblasten der inneren Muskelplatten. und vier von ihnen ausgehende Zellreihen (bei Isopoden - Ligia und Oniscus - bildet J. Nusbaum jederseits fünf innere Teloblasten ab; ich fand aber bei Onisciden jederseits nur vier, ganz ebenso wie PATTEN für Cymothoa).

2) Neue Beiträge zur Embryologie der Anneliden. I. Zeitschr. f. wiss. Zool. Bd. 50 . 
Theilungen zu sehen sind (Fig. 4 und 6); beide Figuren betreffen die Neuralreihe (Reihe I), und zwar liegt die eine Mitose in der Zelle 5, die andere in der Zelle 7. Dies ist das ganze Beobachtungsmaterial; hieraus bestimmte Schlüsse ziehen zu wollen, wäre verfriiht; doch ist es vielleicht wahrscheinlich, dass die Theilungs. potenz dieser Teloblasten ea. $6(5-7) \mathrm{mal}$ so groß ist wie die der kleineren Zellen; damit würde auch die Zunahme der letzteren an Größe, bis sie in die betreffende Region gelangt sind, übereinstimmen ${ }^{1}$. Doch hieriber müssen weitere Untersuchungen den Entscheid bringen.

\section{Criodrilus, innere Keimstreifen (innere Muskelplatten).}

Schon HatscheK $\left.{ }^{2}\right)$ macht ïber das Wachsthum dieser Gebilde folgende Bemerkung: "In der Mesodermzellenreihe sieht man die kleinste Zelle am Hinterende unmittelbar an der Urzelle liegen und. beobachtet eine kontinuirliche Steigerung der Größe der Zellen nach vorn zu. Erst dort, wo der Mesodermstreif mehrreihig wird, werden die Zellen plötzlich kleiner. Das rïhrt daher, dass die kleinen, von der Urzelle aus durch Theilung entstandenen Zellen zuerst heranwachsen, ohne sich zn theilen; wenn endlich ihre Theilung erfolgt, so geschieht dies in der Längsrichtung, so dass dadurch der Mesodermstreif sogleich mehrreihig wird.

Da mir schon lange Criodritus als ein vorzügliches Objekt für das Studium dieser Verhältnisse bekannt war, nahm ich wieder mein altes, im Jahre 1887 zum Zweck anderer Studien präparirtes Material vor und untersuchte die Hinterenden von 56 inneren Muskelplatten oder *Mesodermstreifen . Diese umfassen nur wenige Stadien, namentlich die den Fig. 16 und 17 von HaTscueK entsprechenden; dagegen fehlten mir ganz und gar die jüngeren Phasen.

Die Untersuchung ergab, dass die oben angefuihrten Sätze HatscheK's im Allgemeinen richtig sind. Doch muss Folgendes. hinzngefiugtt werden. Da es für uns sich darum handelte, die Theilungspotenz der Urzelle im Verhältnis zu derjenigen der kleineren Zellen zu bestimmen, so war es zunächst nothwendig zu untersuchen,

1) In den Abbildungen von E. B. WiLson (Journ. of Morph. Vol. 1 u. 3) und von VeJdorskx́ (Entwickelnngsgesch. Untersuchungen. Prag. 1888-1892) finden sich Mitosen nur weiter vorn im Keimstreifen.

2) Studien über Entwickelungsgesch. der Anneliden. Arbeiten a. d. Zool. Inst. Wien. Bd. 1. 1878, pag. 17-18 (293-294). 
ob die Mitosen in den kleineren Zellen in einer bestimmten Entfernung von der Urzelle auftreten. Und zwar ergab diese Untersuchung ein recht positives Resultat. Unter den 56 zu Gesicht gekommenen Streifen wurden die Urzellen $12 \mathrm{mal}$ in Theilung gefunden; in den jüngsten neun Zellen, die unmittelbar vor der Urzelle lagen, fanden sich nie Theilungen; dagegen wurde die Zelle 10 zweimal, die Zelle 11 viermal und die Zelle 12 zweimal in Theilung: gefunden. Ich glaube, dass dies schwerlich auf einer Zufälligkeit beruhen kann, sondern dass der Befund einfach so zu erklären ist, dass in den ersten neun Zellen iberhaupt keine Theilungen auftreten, sondern dass sie erst in der Region der Zellen 10-12 stattfinden. Daraus wiurde wieder folgen, dass die Theilungspotenz der Urzelle (jedenfalls in den von mir untersuchten Stadien) etwa $10 \mathrm{mal}$ so groß, wie die der kleineren Zellen wäre, gewöhnlich sogar $>10$; denn während des Ruhezustandes jeder kleinen Zelle (von dem Augenblick, in dem sie abgeschniurt wurde, bis zu ihrer ersten äqualen Theilung) hat die Urzelle neun (resp. zehn oder elf) Zellen gesprosst. Dieses wird auch dadurch wahrscheinlich gemacht, dass ganz allgemein vor der in Theilung begriffenen Zelle die Anordnung zweireihig, hinter ihr einreihig ist; dies stimmt auch mit der Theilungsrichtung iberein (vgl. Taf. XIX Fig. 9); ist keine Zelle in Theilung begriffen, so beginnt doch die zweireihige Anordnung in den weitaus häufigsten Fällen in der Region der Zellen 11 und 12. Auch ist $\mathrm{ja}$, wie schon ron HatscheK hervorgehoben, meistens sehr deutlich zu erkennen, wie die Zellen von der Urzelle nach der erwähnten Region an Größe zunehmen und dann wieder kleiner werden.

Die erwähnte Anordnung kann doch nicht immer mit solcher Deutlichkeit erkannt werden. Bisweilen stehen die Zellen schon sehr nabe an ihrer Ursprungsstelle (bei der Urzelle) so dicht, dass ihre Anordnung eine zweireihige genannt werden muss; aber niemals traf ich, wie gesagt, Theilungen vor der Zelle 10 an. Die näher an der Urzelle auftretende Zweireihigkeit beruht einfach auf starkem Aneinandergedrängtsein der kleineren Zellen, wohl in Folge starken Drucks von rorn und ron hinten. Auch das umgekehrte Extrem kann gefunden werden und ist dafür der in Fig. 10 dargestellte Fall sehr bezeichnend. Die Zelle 12 ist hier in Theilung und zwar, wie gewöhnlich, in Längstheilung begriffen; da aber gerade an dieser Stelle eine starke Biegung des Streifens stattfindet, wird dieser nicht gleich vor der Zelle 12 zweireihig, sondern 
die folgenden vier Zellen liegen noch in einer Reihe angeordnet, so dass die Zweireihigkeit hier erst mit der siebzehnten Generation anfängt.

In den unmittelbar vor der Region der Zellen 10-12 liegenden Zellen fand ich nie Mitosen; erst etwas weiter rorn treten sie wieder auf; doch vermochte ich wegen der Schwierigkeiten, welche die Anordnung der Zellen hier der Erkenntnis ihrer Generationenfolge darbietet, nicht die Gesetzmäßigkeit aufzudecken.

Also lassen sich aus den obigen Beobachtungen nur folgende Schlïsse ziehen: die erste Theilung der kleineren Zellen ist eine Längstheilung (in Übereinstimmung mit Hatschek) und: die Theilungspotenz der Urzelle ist etwa $10 \mathrm{mal}$ so groB, wie die der kleineren (gewöhnlich $>10$ mal).

Kopenhagen, Mai 1895.

\section{Erklärung der Abbildungen.}

\section{Tafel XIX.}

Fig. 1-3. Armadillidium vulgare.

Fig. 1. Stück des hintersten Theils des voll ausgebildeten Keimstreifens. F. Oc. 1 (ZEISS). $M$ der mediane Teloblast, $m$ die aus ihm entspringende Zellreihe (ebenso in dev anderen Figuren).

Fig. 2. Stück des hinteren Theils des Keimstreifens in einem jüngeren Stadium mit nur sechs Querreihen von Zellen. F. Oc. 1.

Fig. 3. Zwei Teloblasten mit den aus ihnen entspringenden Zellreihen. Die rechte derselben liegt der Mittellinie näher als die linke. F. Oc. 1.

\section{Fig. 4-5. Oniscus murarius.}

Fig. 4. Stïck des hinteren Theils des fertig ausgebildeten Keimstreifens. D. 0c.1. Fig. 5. Die drei äußersten Teloblasten mit den von ihnen gesprossten Zellreihen. D. Oc. 1. Die rechts gelegene ist der Medianlinie am nächsten. In $a$ ist eine Abstraktion des senkrechten Längsschnitts der mittleren der drei Reihen gegeben.

$$
\text { Fig. 6-7. Mysis sp. }
$$

Fig. 6. Stück des hinteren Theils des Keimstreifens im älteren Nuuplius-Stadium (mit umgebogenem Schwanze). F. Oc. 1.

Fig. 7. Ein ebensolches Präparat von dem Keimstreifen des jüngeren Nauplius. D. Oc. 1.

\section{Fig. 8. Gammarus pulex.}

Stïck des hinteren Theils des Keimstreifens eines Stadiums, in welchem der Keimstreifen schräg über das Ei verläuft (Fig. 3 meiner frïheren Arbeit). D. 0c. 1.

Fig. 9-10. Criodrilus lacuum.

Fig. 9. Innerer Myoblast (Urmesodermzelle) mit dem hinteren Theil der von ihr gesprossten Zellreihe in dem Stadium Fig. 16 von HatSCHEK. F. Oc. 1. Fig. 10. Ein ebensolches Präparat in dem Stadium Fig. 17 von HATSchex. F. Oc. 1. 